\title{
Strategy-Proofness And the Tops-Only Property
}

by

\author{
John A. Weymark
}

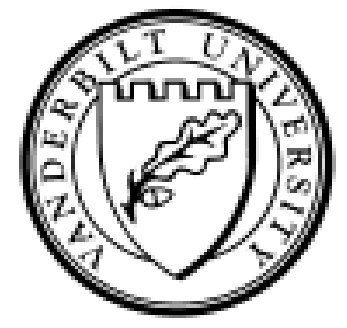

Working Paper No. 04-W09

April 2004

\section{DEPARTMENT OF ECONOMICS \\ VANDERBILT UNIVERSITY \\ NASHVILLE, TN 37235}

www.vanderbilt.edu/econ 


\title{
Strategy-Proofness and the Tops-Only Property
}

\author{
JOHN A. WEYMARK \\ Department of Economics, Box 1819, Station B, \\ Vanderbilt University, Nashville, TN 37235, U.S.A. \\ (e-mail: john.weymark@vanderbilt.edu)
}

April 14, 2004

Revised: April 27, 2004

\begin{abstract}
A social choice function satisfies the tops-only property if the chosen alternative only depends on each person's report of his most-preferred alternatives on the range of this function. On many domains, strategyproofness implies the tops-only property, provided that the range of the social choice function satisfies some regularity condition. The existing proofs of this result are model specific. In this article, a general proof strategy is proposed for showing that a strategy-proof social choice function satisfies the tops-only property when everyone has the same set of admissible preferences.
\end{abstract}

Keywords and Phrases: Social choice; strategy-proofness; option sets; tops-only property.

JEL Classification Numbers: D71, D82. 


\section{Introduction}

One of the main lessons of the literature on strategy-proof social choice is that a social decision procedure must ignore most of the information about individual preferences, otherwise it is sometimes possible for someone to manipulate the outcome by misreporting his preferences. A social choice function satisfies the tops-only property if the chosen alternative only depends on each person's report of his most-preferred alternatives on the range of this function. On many domains, strategy-proofness implies the tops-only property, provided that the range of the social choice function satisfies some regularity condition. In his survey of strategy-proof social choice, Sprumont (1995, p. 77) says that: "Proving this fact constitutes a key step in many papers in the literature. Unfortunately, the proofs remain model specific and are often quite complicated."

In this article, I propose a proof strategy for showing that a strategyproof social choice function satisfies the tops-only property. This methodology presupposes that everyone has the same set of admissible preferences on the set of alternatives. This assumption would not be satisfied if there are private goods and individuals only care about their own consumption. My methodology also assumes that for each admissible preference, there is a unique most-preferred alternative on the range of the social choice function. When this condition is not satisfied, it is nevertheless possible in some circumstances to characterize the structure of a strategy-proof social choice function by first restricting attention to a subdomain in which this property is satisfied. See, for example, Barberà and Peleg (1990) and Le Breton and Weymark (1999). The proof strategy proposed here is based on the strategy employed by Le Breton and Weymark (1999) to prove that the tops-only property is implied by strategy-proofness when there is a product set of alternatives, preferences are continuous and separable with a unique best alternative, and the range of the social choice function is suitably restricted. Their proofs (and a fortiori the proofs presented here) make use of arguments first developed by Barberà and Peleg (1990) and Barberà and Jackson (1994).

My approach to proving that strategy-proofness implies the tops-only property employs the option-set methodology introduced by Barberà (1983), Laffond (1980), and Satterthwaite and Sonnenschein (1981). The option set facing a subgroup of the population is the set of alternatives that are feasible for a social choice function given the reported preferences of the 
rest of the population. The use of option sets by Barberà and Peleg (1990) to prove a version of the Gibbard (1973)-Satterthwaite (1975) Theorem in which preferences are required to be continuous is largely responsible for the revival of interest in strategy-proof social choice over the past decade and a half. ${ }^{1}$ For surveys of this literature, see Barberà (2001) and Sprumont (1995).

I illustrate my proof strategy with two examples. In my first example, the set of alternatives is a subset of the real line, the domain of the social choice function consists of all the single-peaked preferences on this set, and the range of the social choice function is an interval. The analysis of strategyproofness on the domain of single-peaked preferences was initiated by the seminal article of Moulin (1980). Versions of the tops-only result for this domain have been established by Barberà and Jackson (1994), Barberà, Gul, and Stacchetti (1993), and Ching (1997), but their proofs differ from mine in a number of respects. ${ }^{2}$ In my second example, the set of alternatives is a metric space and the domain is the set of continuous preferences with unique best alternatives on the range of the social choice function. This is the domain considered by Barberà and Peleg (1990). They do not explicitly prove a topsonly result for their domain, but the tops-only property is implied by their main theorem.

I do not claim that my approach to proving that the tops-only property is implied by strategy-proofness applies for all domains in which all preferences have unique best alternatives on the range of the social choice function. Indeed, it is clear that the domain must be reasonably rich in order for my arguments to apply. However, my proof strategy does work on a number of domains that have been considered in the literature. Further examples of such domains are described in my concluding comments. Thus, the proof strategy proposed here provides a unified perspective from which to view many of the existing tops-only results. Hopefully, it will also facilitate the development of tops-only theorems on domains that have not previously been considered.

\footnotetext{
${ }^{1}$ The Gibbard (1973)-Satterthwaite (1975) Theorem shows that a strategy-proof social choice function is dictatorial when the individual preferences are unrestricted and the range of the social choice function contains at least three alternatives. An individual is a dictator if for all preference profiles in the domain of the social choice function, the chosen alternative maximizes this person's preference on the range of the social choice function.

${ }^{2} \mathrm{~A}$ tops-only theorem for the related problem of allocating a divisible private good among individuals with single-peaked preferences over own consumption had previously been established by Sprumont (1991).
} 
In the next section, I set out the notation and basic definitions that are employed in subsequent sections. In Section 3, I review a number of theorems that identify some properties that strategy-proof social choice functions must exhibit when individuals have the same set of admissible preferences. My proof strategy is used in Section 4 to prove a tops-only theorem for singlepeaked preferences. The analogous theorem for continuous preferences with unique best alternatives on the range of the social choice function is established in Section 5. Some concluding remarks are provided in Section 6.

\section{Notation and basic definitions}

The set of individuals is $N=\{1, \ldots, n\}$, where $n$ is finite. The set of alternatives is $A$. In order for the social choice problem to be nontrivial, it is assumed throughout that $|A| \geq 2$. Further assumptions about $A$ will be made in subsequent sections.

An ordering $R$ on $A$ is a reflexive, complete, and transitive binary relation. The corresponding strict preference and indifference relations are denoted by $P$ and $I$, respectively. The set of all orderings of $A$ is $\mathcal{R}$. Preferences may be restricted a priori. The domain of individual preferences is $\mathcal{D} \subseteq \mathcal{R}$. Each individual $h \in N$ is assumed to have a preference ordering $R^{h} \in \mathcal{D}$. A profile is an $n$-tuple of individual preference orderings $\mathbf{R}=\left(R^{1}, \ldots, R^{n}\right)$. The set of admissible profiles is $\mathcal{D}^{n}$.

By assuming that everyone has the same individual preference domain, I am implicitly assuming that the alternatives are public in nature. If there are private goods and individuals only care about their own consumption, then the individual preference domains would need to be person-specific. The assumption that the set of admissible preference profiles is the Cartesian product of identical individual preference domains is used in a fundamental way in establishing most of the theorems presented here.

A social choice function is a function $f: \mathcal{D}^{n} \rightarrow A$. The range of $f$ is

$$
A^{f}=\left\{x \in A \mid f(\mathbf{R})=x \text { for some } \mathbf{R} \in \mathcal{D}^{n}\right\} .
$$

If there are resource constraints that preclude some of the alternatives in $A$ from being feasible, as in Barberà, Massó, and Neme (1997), then we also need to introduce the set of feasible alternatives $Z \subseteq A$. Preferences would still be defined on all of $A$, but the range would then be a subset of $Z$. In this article, I assume that $A=Z$ and, henceforth, do not distinguish between $A$ and $Z$. 
The vector $\mathbf{R}^{H}=\left(R^{h}\right)_{h \in H}$ denotes the subprofile of preferences of the individuals in $H$, where $\emptyset \subset H \subset N .^{3}$ When I want to focus on the subgroups $H$ and $-H$ (the complement of $H$ ), a profile is written as $\mathbf{R}=\left(\mathbf{R}^{H} ; \mathbf{R}^{-H}\right)$. For the social choice function $f$, the option set generated by $\mathbf{R}^{H}$ is

$$
O_{-H}^{f}\left(\mathbf{R}^{H}\right)=\left\{x \in A \mid x=f\left(\mathbf{R}^{H} ; \mathbf{R}^{-H}\right) \text { for some } \mathbf{R}^{-H} \in \mathcal{D}^{n-|H|}\right\} .
$$

$O_{-H}^{f}\left(\mathbf{R}^{H}\right)$ is the set of alternatives that are attainable given that the individuals in $H$ have reported the subprofile $\mathbf{R}^{H}$.

Given a subprofile $\mathbf{R}^{H} \in \mathcal{D}^{|H|}$, we can use $f$ to define an $(n-|H|)$-person social choice function $g: \mathcal{D}^{n-|H|} \rightarrow A$ by setting

$$
g\left(\mathbf{R}^{-H}\right)=f\left(\mathbf{R}^{H} ; \mathbf{R}^{-H}\right) \text { for all } \mathbf{R}^{-H} \in \mathcal{D}^{n-|H|} .
$$

The option set $O_{-H}^{f}\left(\mathbf{R}^{H}\right)$ is simply the range of $g$. If $|H|=n-1, g$ is a oneperson social choice function. Although there is no social choice problem if there is only one individual, in order to identify some of the properties of option sets generated by the preferences of all but one individual, it is sometimes necessary to consider one-person social choice functions. Except when this is the case, it is assumed that $n \geq 2$.

A social choice function $f$ is manipulable by person $h \in N$ at the profile $\mathbf{R} \in \mathcal{D}^{n}$ via $\bar{R}^{h} \in \mathcal{D}$ if $f\left(R^{1}, \ldots, R^{h-1}, \bar{R}^{h}, R^{h+1}, \ldots, R^{n}\right) P^{h} f(\mathbf{R})$. If there is no individual $h \in N$, no profile $\mathbf{R} \in \mathcal{D}^{n}$, and no preference $\bar{R}^{h} \in \mathcal{D}$ such that $f$ is manipulable by person $h$ at $\mathbf{R}$ via $\bar{R}^{h}$, then $f$ is strategy-proof.

For any $R \in \mathcal{R}$ and any nonempty set $S \subseteq A$, the top set of $R$ in $S$ is

$$
\tau(R, S)=\{x \in S \mid x R y \text { for all } y \in S\} .
$$

The top sets considered in subsequent sections all have the property that they contain exactly one alternative. A social choice function $f$ has the tops-only property if $f(\mathbf{R})=f(\overline{\mathbf{R}})$ for all $\mathbf{R}, \overline{\mathbf{R}} \in \mathcal{D}^{n}$ for which $\tau\left(R^{h}, A^{f}\right)=\tau\left(\bar{R}^{h}, A^{f}\right)$ for all $h \in N$. That is, the only information about preferences that $f$ is sensitive to is each person's top set on the range of $f$.

In some of the literature, the tops-only property is defined in terms of the top sets for $A$, not $A^{f}$. However, this alternative definition has typically been adopted for problems in which the two definitions coincide. This will be the case if either (i) $A=A^{f}$ or (ii) $A \neq A^{f}$ and the top set on $A^{f}$ is uniquely determined by the top set on $A$ for all admissible preferences. In the former case, the social choice function is said to be nonimposed. The latter case applies to the problem considered in Section 4.

\footnotetext{
${ }^{3}$ If $H=\{h\}$, the braces are omitted.
} 


\section{General results}

In this section, I review a number of basic properties of strategy-proof social choice functions that hold quite generally when the domain is the Cartesian product of a common individual preference domain. My discussion is based on Section 2 of Le Breton and Weymark (1999). Their theorems are more general than needed here, so, in most cases, I sacrifice some of this generality in order to make the presentation more accessible. With the exception of Proposition 1, versions of Le Breton and Weymark's theorems had previously been established for a number of particular specifications of $A$ and $\mathcal{D}$. Throughout this section, it is assumed that $f: \mathcal{D}^{n} \rightarrow A$ is a strategy-proof social choice function.

The first four propositions in this section make no assumptions about the structure of the set of alternatives and the domain of the social choice function other than my maintained assumption that the domain is the Cartesian product of a common individual preference domain.

Proposition 1 establishes that any admissible preference must have a welldefined maximum on $A^{f}$.

Proposition 1. Suppose that $n \geq 1$. If a social choice function $f: \mathcal{D}^{n} \rightarrow A$ is strategy-proof, then for all $R \in \mathcal{D}, \tau\left(R, A^{f}\right) \neq \emptyset$.

The next proposition is a unanimity result. It shows that alternative $x$ must be chosen if everybody agrees that $x$ is uniquely best on $A^{f}$.

Proposition 2. Suppose that $n \geq 1$ and that $f: \mathcal{D}^{n} \rightarrow A$ is a strategy-proof social choice function. If there exists an $x \in A^{f}$ such that $\tau\left(R^{h}, A^{f}\right)=\{x\}$ for all $h \in N$, then $f(\mathbf{R})=x$.

Recall that the option set $O_{-H}^{f}\left(\mathbf{R}^{H}\right)$ is the range of the social choice function $g$ defined in (1). Note that if $f$ is strategy-proof, then so is $g$. By applying Proposition 2 to $g$, it follows that if the individuals not in $H$ agree that $x$ is uniquely best on this option set, then it must be the social choice.

Proposition 3. Suppose that $n \geq 2$ and that $f: \mathcal{D}^{n} \rightarrow A$ is a strategy-proof social choice function. For all nonempty $H \subset N$ and all $\mathbf{R}^{H} \in \mathcal{D}^{|H|}$, if $\tau\left(R^{h}, O_{-H}^{f}\left(\mathbf{R}^{H}\right)\right)=\{x\}$ for all $h \notin H$, then $f(\mathbf{R})=x$.

Another implication of Proposition 2 is that if a subgroup agrees that $x$ is uniquely best on $A^{f}$, then $x$ must be in the option set that their preferences generate. 
Proposition 4. Suppose that $n \geq 2$ and that $f: \mathcal{D}^{n} \rightarrow A$ is a strategy-proof social choice function. For all nonempty $H \subset N$, if $\tau\left(R^{h}, A^{f}\right)=\{x\}$ for all $h \in H$, then $x \in O_{-H}^{f}\left(\mathbf{R}^{H}\right)$.

For the final two propositions in this section, it is assumed that $A$ is a subset of a metric space. ${ }^{4}$ In many applications of these results, $A$ is in fact a subset of a Euclidean space. It is also assumed that the domain of $f$ satisfies a regularity condition. A social choice function $f: \mathcal{D}^{n} \rightarrow A$ has a regular domain if for all $x \in \bar{A}^{f}$, there exists a continuous preference $R \in \mathcal{D}$ such that $\tau\left(R, \bar{A}^{f}\right)=\{x\} .^{5}$ In other words, for any alternative $x$ in the closure of the range $\bar{A}^{f}$, there is a continuous preference in the individual preference domain that is uniquely maximized on $\bar{A}^{f}$ at $x$.

This domain regularity condition is satisfied in the problems considered in Sections 4 and 5, as well as in many other problems considered in the literature. However, there are problems of economic interest that satisfy my other domain restrictions without being regular. For example, suppose that $A$ is the set of lotteries generated by three or more certain alternatives and that $\mathcal{D}$ is the set of von Neumann-Morgenstern preferences on $A$. If the social choice function is nonimposed, then the domain is not regular because only a vertex of $A$ can be a unique maximizer of a von Neumann-Morgenstern preference on $A{ }^{6}$

Proposition 5 demonstrates that the range of $f$ must be a closed set whenever $A$ is a subset of a metric space, provided that the domain regularity condition is satisfied.

\footnotetext{
${ }^{4}$ These results hold more generally for any first-countable topological space.

${ }^{5} \mathrm{~A}$ preference $R \in \mathcal{R}$ is continuous if for all $x \in A,\{y \in A \mid y R x\}$ and $\{y \in A \mid x R y\}$ are both closed sets.

${ }^{6}$ Gibbard (1977), Ehlers, Peters, and Storcken (2002), and Dutta, Peters, and Sen (2002), among others, model probabilistic social choice using a probabilistic decision scheme, rather than a social choice function. A probabilistic decision scheme assigns a probability measure over the set of certain outcomes $A$ to each admissible profile of preferences on A. Ehlers, Peters, and Storcken (2002) and Dutta, Peters, and Sen (2002) have established tops-only theorems using this framework. A probabilistic decision scheme $p$ can be reformulated as a social choice function $f$ for the set of probability measures $\Delta(A)$ on $A$ if the domain of $f$ is permitted to include profiles of incomplete preference relations on $\Delta(A)$. The function $f$ associated with $p$ is defined by ordinally extending each admissible preference $R$ on $A$ to an incomplete preference $Q(R)$ on $\Delta(A)$ using first-degree stochastic dominance with respect to the upper contour sets of $R$. The top of $Q(R)$ on $\Delta(A)$ is the probability measure that assigns the top of $R$ on $A$ probability 1 . Thus, the domain of $f$ cannot be regular if, as in the articles cited above, random dictatorships are in the range of the social choice function and the tops of the preferences on $A$ are unique.
} 
Proposition 5. Suppose that $n \geq 1$ and that $A$ is a subset of a metric space. If $f: \mathcal{D}^{n} \rightarrow A$ is a strategy-proof social choice function with a regular domain, then $A^{f}$ is closed.

This result is easily established by observing that if $A^{f}$ is not closed, then for any $x \in \bar{A}^{f} \backslash A$, any $y \in A^{f}$, and any continuous preference $R \in \mathcal{D}$ for which $\tau\left(R, \bar{A}^{f}\right)=\{x\}$, there must be alternatives close to $x$ in $A$ that are strictly preferred to $y$ by $R$. This implies that the top set of $R$ on $A^{f}$ is empty, contradicting Proposition 1.

Because the option set $O_{-H}^{f}\left(\mathbf{R}^{H}\right)$ is the range of the social choice function $g$ defined in (1) and because strategy-proofness and regularity are properties that $g$ inherits from $f$, it follows from Proposition 5 that option sets are closed when $A$ is a subset of a metric space and the domain is regular.

Proposition 6. Suppose that $n \geq 2$ and that $A$ is a subset of a metric space. If $f: \mathcal{D}^{n} \rightarrow A$ is a strategy-proof social choice function with a regular domain, then for all nonempty $H \subset N$ and all $\mathbf{R}^{H} \in \mathcal{D}^{|H|}, O_{-H}^{f}\left(\mathbf{R}^{H}\right)$ is closed.

\section{Single-Peaked Preferences}

In this section, $A$ is assumed to be a subset of the real line $\mathbb{R}$ and preferences are assumed to be single-peaked on this set. Depending on the application, $A$ could be a discrete or a connected set. For example, $A$ could be a finite set of candidates for election, arrayed on a left-right ideological spectrum. Alternatively, $A$ could be the nonnegative line $\mathbb{R}_{+}$, with $x \in A$ interpreted as being the quantity of some public good.

It is useful to have a definition of an interval of $A$ that applies whether or not $A$ is itself an interval of $\mathbb{R}$. A subset $S$ of $A \subseteq \mathbb{R}$ is an interval of $A$ if $[x, y] \cap A \subseteq S$ whenever $x, y \in S$. In other words, for any two points in $S$, all the points in $A$ lying between $x$ and $y$ are also in $S$. The closed interval of $A$ containing all points between $x$ and $y$ is denoted $x y$.

A preference $R$ on $A$ is single-peaked if there exists an alternative $\pi(R) \in$ $A$ such that $\pi(R) P x P y$ whenever $x, y \in A$ and $y<x<\pi(R)$ or $\pi(R)<$ $x<y$. The alternative $\pi(R)$ is the peak of the preference $R$. The set of single-peaked preferences on $A$ is $\mathcal{S}$.

It is assumed that the social choice function $f$ is defined on the set of all profiles of single-peaked preferences $\mathcal{S}^{n}$. It is also assumed that the range $A^{f}$ is an interval of $A$. Note that $f$ has a regular domain, so by Propositions 5 and 6 , the range and all option sets of $f$ must be closed if $f$ is strategy-proof. 
For all $R \in \mathcal{S}$, the top set $\tau(R, S)$ is uniquely defined by the peak $\pi(R)$ if $S$ is a closed interval of $A$. Suppose that $x$ (resp. $y$ ) is the smallest (resp. largest) point in $S$. We then have three cases. (i) If $\pi(R) \in S$, then $\tau(R, S)=\{\pi(R)\}$. (ii) If $\pi(R)<x$, then $\tau(R, S)=\{x\}$. (iii) If $\pi(R)>y$, then $\tau(R, S)=\{y\}$. Thus, if two preferences in $\mathcal{S}$ have the same peak on $A$, they also have the same top set on the range $A^{f}$ if the range is a closed interval of $A$.

To show that strategy-proofness implies the tops-only property on the domain of single-peaked preferences when the range of the social choice function is an interval of $A$, I first establish a number of intermediate results. The first of these results, Lemma 1, shows that when a set of individuals $H$ agrees that some alternative is uniquely best on the range of the social choice function, then how these individuals rank the other alternatives is irrelevant in determining the option set that their preferences generate. In effect, Lemma 1 shows that option sets satisfy a tops-only property when there is agreement among the individuals in $H$ as to which alternative is best on the range. In the particular case in which there is only one person in $H$, Lemma 1 says that the option set generated by this person's preference only depends on his most-preferred alternative on the range, provided that there is only one such alternative. The limited form of a tops-only property for option sets established in Lemma 1 when $|H| \geq 2$ is used in a fundamental way in the proof of Lemma 2.

Lemma 1. Suppose that $n \geq 2, A \subseteq \mathbb{R}$, and $A^{f}$ is an interval of $A$. If $f: \mathcal{S}^{n} \rightarrow A$ is a strategy-proof social choice function, then for all nonempty $H \subset N$ and all $\mathbf{R}^{H}, \overline{\mathbf{R}}^{H} \in \mathcal{S}^{|H|}$ for which $\tau\left(R^{h}, A^{f}\right)=\tau\left(\bar{R}^{k}, A^{f}\right)$ for all $h, k \in H, O_{-H}^{f}\left(\mathbf{R}^{H}\right)=O_{-H}^{f}\left(\overline{\mathbf{R}}^{H}\right)$.

Proof. The lemma is trivial if $\left|A^{f}\right|=1$, so suppose that $\left|A^{f}\right| \geq 2$. Without loss of generality, we can suppose that $H=\{1, \ldots, k\}$ with $1 \leq k<n$. Consider the sequence of subprofiles:

$$
\begin{gathered}
\mathbf{R}^{H, 0}=\left(R^{1}, \ldots, R^{k}\right)=\mathbf{R}^{H}, \\
\mathbf{R}^{H, 1}=\left(\bar{R}^{1}, R^{2}, \ldots, R^{k}\right), \\
\vdots \\
\mathbf{R}^{H, k-1}=\left(\bar{R}^{1}, \ldots, \bar{R}^{k-1}, R^{k}\right), \\
\mathbf{R}^{H, k}=\left(\bar{R}^{1}, \ldots, \bar{R}^{k}\right)=\overline{\mathbf{R}} .
\end{gathered}
$$


To establish the lemma, it is sufficient to show that

$$
O_{-H}^{f}\left(\mathbf{R}^{H, h-1}\right)=O_{-H}^{f}\left(\mathbf{R}^{H, h}\right)
$$

for all $h \in H$.

On the contrary, suppose that there exists an $\bar{h} \in H$ such that (2) does not hold for $h=\bar{h}$. Without loss of generality, we can suppose that there exists a $y \in O_{-H}^{f}\left(\mathbf{R}^{H, \bar{h}-1}\right)$ with $y \notin O_{-H}^{f}\left(\mathbf{R}^{H, \bar{h}}\right)$.

By assumption, $\tau\left(R^{h}, A^{f}\right)=\tau\left(\bar{R}^{k}, A^{f}\right)$ for all $h, k \in H$. Call this common value $x$. Without loss of generality, we can suppose that $x<y$. By Proposition $4, x \in O_{-H}^{f}\left(\mathbf{R}^{H, \bar{h}-1}\right) \cap O_{-H}^{f}\left(\mathbf{R}^{H, \bar{h}}\right)$. By Proposition $6, O_{-H}^{f}\left(\mathbf{R}^{H, \bar{h}}\right)$ is a closed set. Hence, in $\overline{x y}$ there is a unique closest point to $y$ in $O_{-H}^{f}\left(\mathbf{R}^{H, \bar{h}}\right)$. Call this point $z .^{7}$ This construction is illustrated in Figure 1 for the case in which $A=\mathbb{R}$. Because there is an open neighbourhood of $y$ not contained in $O_{-H}^{f}\left(\mathbf{R}^{H, \bar{h}}\right)$, we can find a preference $R^{*}$ in $\mathcal{S}$ with peak at $y$ that is maximized at $z$ in $O_{-H}^{f}\left(\mathbf{R}^{H, \bar{h}}\right)$. By Proposition 3 , we have $f\left(\mathbf{R}^{H, \bar{h}-1} ; R^{*}, \ldots, R^{*}\right)=y$ and $f\left(\mathbf{R}^{H, \bar{h}} ; R^{*}, \ldots, R^{*}\right)=z$. But $z P^{\bar{h}} y$ because $z$ lies between $x$ (which is $\bar{h}$ 's top alternative on the range) and $y$. Hence, $\bar{h}$ can manipulate $f$ at $\left(\mathbf{R}^{H, \bar{h}-1} ; R^{*}, \ldots, R^{*}\right)$ via $\bar{R}^{\bar{h}}$, contradicting strategy-proofness.

In order to see how the strategy used to prove Lemma 1 can be applied to other domains, it is instructive to summarize the proof strategy without the details that depend on the specific domain used in the lemma. To establish the lemma, there is no loss of generality in supposing that only the preference of one person, say person $\bar{h}$, differs in the two subprofiles $\mathbf{R}^{H}$ and $\overline{\mathbf{R}}^{H}$.

By assumption, $x$ is the uniquely-best alternative on the range of the social choice function $f$ for $\bar{h}$ with either of his two preferences. Furthermore, everyone else in $H$ agrees that $x$ is best on the range. Hence, by Proposition $4, x$ is in the option set facing the rest of the population with either of $\bar{h}$ 's preferences. Contrary to the lemma (and without loss of generality), it is supposed that there is an alternative $y$ that is in the option set when $\bar{h}$ has the preference $R^{\bar{h}}$, but not when he has the preference $\bar{R}^{\bar{h}}$. Because the domain is regular, we know from Proposition 6 that the option set $O_{-H}^{f}\left(\mathbf{R}^{H, \bar{h}}\right)$ is closed.

The next step in the argument shows that there exists an alternative $y \notin$ $O_{-H}^{f}\left(\mathbf{R}^{H, \bar{h}}\right)$ and a preference $R^{*}$ in the domain that satisfies two properties: (i) it is maximized at $y$ and (ii) it is maximized on the option set $O_{-H}^{f}\left(\mathbf{R}^{H, \bar{h}}\right)$

\footnotetext{
${ }^{7}$ If $\left|A^{f}\right|=2, z$ must be equal to $x$ because $x$ is the only alternative in $O_{-H}^{f}\left(\mathbf{R}^{H, \bar{h}}\right)$.
} 
at a unique alternative $z$ that is preferred to $y$ by any preference whose mostpreferred alternative on the range is at $x$. How such a preference is identified is domain specific. For the domain considered in this section, a preference satisfying properties (i) and (ii) can be constructed for any $y \notin O_{-H}^{f}\left(\mathbf{R}^{H, \bar{h}}\right)$. However, on other domains, such a preference may only exist for some y $\notin$ $O_{-H}^{f}\left(\mathbf{R}^{H, \bar{h}}\right)$.

The proof is completed by supposing that everyone not in $H$ has the preference $R^{*}$. Because these individuals agree that $z$ is the best alternative in the option set $O_{-H}^{f}\left(\mathbf{R}^{H, \bar{h}}\right)$, Proposition 3 implies that $z$ must be chosen when $\bar{h}$ reports $\bar{R}^{\bar{h}}$. Similarly, because $y$ is their best alternative in the option set $O_{-H}^{f}\left(\mathbf{R}^{H, \bar{h}-1}\right)$, Proposition 3 implies that $y$ must be chosen when $\bar{h}$ reports $R^{\bar{h}}$. Which option set applies is determined by $\bar{h}$ 's report. By property (ii), we have $z P^{\bar{h}} y$. Hence, $\bar{h}$ can manipulate the outcome by reporting $\bar{R}^{\bar{h}}$ when his true preference is $R^{\bar{h}}$, contradicting strategy-proofness. Therefore, when a group of individuals $H$ agrees that some alternative $x$ is uniquely best on the range, the option set they generate cannot depend on their rankings of the other alternatives.

Other than assuming that the domain is regular, the only other feature of the domain that has been used in the preceding argument is that there is a preference in the domain satisfying properties (i) and (ii) for some $y \notin$ $O_{-H}^{f}\left(\mathbf{R}^{H, \bar{h}}\right)$. Thus, a version of Lemma 1 can be established for any regular domain if for any subprofile $\mathbf{R}^{H}$ in which the individuals in $H$ agree that there is an alternative $x$ that is best on the range and for some $y$ not in the option set generated by this subprofile, there exists a preference in the domain satisfying properties (i) and (ii).

The proof strategy used to establish Lemma 1 is based on the proof used by Barberà and Peleg (1990) to prove a version of this lemma in which $|H|=1$ and the domain is the one considered in the next section. In their proof, the preference $R^{*}$ is chosen so that property (ii) is satisfied with $z=x$ (the best alternative on the range for $R^{\bar{h}}$ ). In general, it is not possible to have $z=x$ with single-peaked preferences.

Throughout this section, it is assumed that the range of the social choice function is an interval. Lemma 2 demonstrates that this property of the range is inherited by any option set generated by a single individual's preference when the domain is the set of single-peaked preferences and the social choice function is strategy-proof.

Lemma 2. Suppose that $n \geq 2, A \subseteq \mathbb{R}$, and $A^{f}$ is an interval of $A$. If 
$f: \mathcal{S}^{n} \rightarrow A$ is a strategy-proof social choice function, then for all $h \in N$ and all $R^{h} \in \mathcal{S}, O_{-h}^{f}\left(R^{h}\right)$ is a closed interval of $A$.

Proof. The proof is trival if $\left|A^{f}\right| \leq 2$, so suppose that $\left|A^{f}\right| \geq 3$. By Proposition 6, $O_{-h}^{f}\left(R^{h}\right)$ is a closed set. Contrary to the lemma, suppose that there exists an $h \in H$ and an $R^{h} \in \mathcal{S}$ such that this option set is not an interval. Hence, there exist $\alpha, \beta, \gamma \in A^{f}$ with $\alpha<\beta<\gamma$ such that $\alpha, \gamma \in O_{-h}^{f}\left(R^{h}\right)$ and $(\alpha, \gamma) \cap O_{-h}^{f}\left(R^{h}\right)=\emptyset$. See Figure 2 for the case in which $A=\mathbb{R}$. Because $A^{f}$ is a closed interval, $\tau\left(R^{h}, A^{f}\right)$ contains a single alternative, say $x$. By Proposition 4, $x \in O_{-h}^{f}\left(R^{h}\right)$. Without loss of generality, we can suppose that $x \geq \gamma$ (otherwise, the roles of $\alpha$ and $\gamma$ can be reversed). Because $R^{h}$ is single-peaked, we thus have $\gamma P^{h} \alpha$.

We can construct a preference $R^{\alpha} \in \mathcal{S}$ with peak at $\beta$ that is maximized on $O_{-h}^{f}\left(R^{h}\right)$ at $\alpha$. Similarly, we can find a preference $R^{\gamma} \in \mathcal{S}$ with peak at $\beta$ that is maximized on $O_{-h}^{f}\left(R^{h}\right)$ at $\gamma$. By Proposition 3, we have $f\left(R^{h} ; R^{\alpha}, \ldots, R^{\alpha}\right)=\alpha$ and $f\left(R^{h} ; R^{\gamma}, \ldots, R^{\gamma}\right)=\gamma$. Note that $\beta \in A^{f}$ because $A^{f}$ is an interval. Hence, by Lemma $1, O_{h}^{f}\left(R^{\alpha}, \ldots, R^{\alpha}\right)=$ $O_{h}^{f}\left(R^{\gamma}, \ldots, R^{\gamma}\right)$. Strategy-proofness implies that $R^{h}$ is maximized at $\alpha$ on $O_{h}^{f}\left(R^{\alpha}, \ldots, R^{\alpha}\right)$ and at $\gamma$ on $O_{h}^{f}\left(R^{\gamma}, \ldots, R^{\gamma}\right)$. Therefore, $\alpha I^{h} \gamma$, contradicting our observation that $\gamma P^{h} \alpha$.

Lemma 2 shows that there is a structural property of the range that is inherited by the option sets generated by a single person's preference when the social choice function is strategy-proof. In this section, this structural property is that the range is an interval. The regularity of the domain ensures that this interval is closed.

For other domains for which a version of Lemma 2 can be established, it is first necessary to identify what structural property to impose on the range of the social choice function $f$. For example, when the set of alternatives is $\mathbb{R}^{m}$ for some $m \geq 2$, Le Breton and Weymark (1999) assume that the range is what they call a coordinate hyperplane. With a coordinate hyperplane, the values for some coordinates are unrestricted, whereas the values of the other components are fixed.

For my proof strategy to apply, it is necessary that each preference $R$ in the domain has a unique maximum on any set of alternatives that satisfies the structural property assumed for the range, and this maximum must only depend on the top of $R$ on the range. This restriction is satisfied here because any single-peaked preference has a unique best alternative $x$ on any closed 
interval of $A$ and the only information about this preference that is needed to identify $x$ is its peak.

Lemma 2 is trivially true for any option set that only contains one alternative because any single point in the range is a closed interval of $A$. However, on other domains, an option set consisting of a single alternative may not satisfy the structural property assumed for the range of the social choice function. On such an option set, any preference necessarily has a unique maximum determined by its top on the range, and that is all that is needed to apply my proof strategy. As a consequence, on some domains, the analogue to Lemma 2 shows that each option set generated by a single person's preference either preserves some structural property of the range or it contains only a single alternative.

The basic proof strategy can be summarized as follows. Contrary to the lemma, assume that there is an individual $h$ and a preference $R^{h}$ in the domain for which the option set $O_{-h}^{f}\left(R^{h}\right)$ generated by $R^{h}$ does not satisfy the structural property stated in the lemma, but which contains at least two alternatives. An implication of this assumption is that there is an alternative $\beta$ satisfying certain properties that is not in the option set $O_{-h}^{f}\left(R^{h}\right)$, but is in the range $A^{f} .8$ For example, in the proof of Lemma $2, \beta$ lies in the smallest interval containing the option set. In general, the location of $\beta$ relative to the option set $O_{-h}^{f}\left(R^{h}\right)$ plays a role in constructing the preferences $R^{\alpha}$ and $R^{\gamma}$ described below, as does the fact (implied by strategy-proofness) that the alternative $x$ that maximizes $R^{h}$ on the range is in this option set.

The proof proceeds by constructing two preferences $R^{\alpha}$ and $R^{\gamma}$ in the domain that are both maximized on the range at $\beta$, but are maximized at distinct points $\alpha$ and $\gamma$ on the option set $O_{-h}^{f}\left(R^{h}\right)$. The closure of the option set, which by Proposition 6 is implied by strategy-proofness and the regularity of the domain, is used in this step of the argument. The preferences $R^{\alpha}$ and $R^{\gamma}$ are chosen so that the alternatives $\alpha$ and $\gamma$ are not indifferent to each other according to the preference $R^{h}$. Without loss of generality, these alternatives can be labelled so that $\gamma P^{h} \alpha$. For example, in the proof of Lemma 2, because the peak of the single-peaked preference $R^{h}$ on the range is $x$, if $\alpha$ and $\gamma$ are chosen as in Figure 2, it is necessarily the case that $R^{h}$ does not regard $\alpha$ and $\gamma$ as being indifferent.

When everyone other than $h$ shares the preference $R^{\alpha}$, they agree that $\alpha$ is

\footnotetext{
${ }^{8}$ For the domain considered in Section $5, \beta$ can be any alternative not in the option set, but this is atypical.
} 
the uniquely-best alternative on the option set $O_{-h}^{f}\left(R^{h}\right)$. Proposition 3 then implies that $\alpha$ must be chosen for the profile $\left(R^{h} ; R^{\alpha}, \ldots, R^{\alpha}\right)$. Similarly, $\gamma$ must be chosen for the profile $\left(R^{h} ; R^{\gamma}, \ldots, R^{\gamma}\right)$.

Consider a profile $\overline{\mathbf{R}}$ for which person $h$ has the preference $\bar{R}^{h}$ and everyone else has the preference $\bar{R}$. Suppose that $\bar{R}$ has a unique maximum on the option set $O_{-h}^{f}\left(\bar{R}^{h}\right)$. Proposition 3 implies that the social choice $f(\overline{\mathbf{R}})$ must simultaneously (i) maximize $\bar{R}^{h}$ on the option set generated by the subprofile $\overline{\mathbf{R}}^{-h}$ and (ii) maximize $\bar{R}$ on the option set generated by $\bar{R}^{h}$. Thus, $\alpha$ must maximize $R^{h}$ on the option set $O_{h}^{f}\left(R^{\alpha}, \ldots, R^{\alpha}\right)$ and $\gamma$ must maximize $R^{h}$ on the option set $O_{h}^{f}\left(R^{\gamma}, \ldots, R^{\gamma}\right)$.

Next, Lemma 1 is applied for a subgroup $H$ consisting of everyone but person $h$. Because the tops of $R^{\alpha}$ and $R^{\gamma}$ on the range are the same, the option sets $O_{h}^{f}\left(R^{\alpha}, \ldots, R^{\alpha}\right)$ and $O_{h}^{f}\left(R^{\gamma}, \ldots, R^{\gamma}\right)$ are identical. Because both $\alpha$ and $\gamma$ maximize $R^{h}$ on this set, we must have $\alpha I^{h} \gamma$, contradicting the fact that $\gamma P^{h} \alpha$, which completes the proof.

If it is not possible to find preferences $R^{\alpha}$ and $R^{\gamma}$ such that $\neg\left(\alpha I^{h} \gamma\right)$, it is nevertheless possible to employ the same basic proof strategy if there is a preference $\tilde{R}^{h}$ in the domain with the same most-preferred alternative on the range as $R^{h}$ for which such preferences exist. By Lemma $1, R^{h}$ and $\tilde{R}^{h}$ generate the same option set, so the preceding proof applies with $\tilde{R}^{h}$ substituting for $R^{h}$.

The proof strategy used to establish Lemma 2 is based on the proof used by Le Breton and Weymark (1999) to prove the analogous lemma in their article. The main difficulties encountered in adapting this result to other domains are (i) identifying the structural property of the range that is preserved by non-singleton option sets generated by the preferences of single individuals and (ii) constructing the preferences $R^{\alpha}$ and $R^{\gamma}$. As Le Breton and Weymark's article demonstrates, the latter constructions might be quite involved.

Lemma 3 extends Lemma 2 by showing that any option set, not just those generated by a single individual's preference, must be an interval if the range satisfies this property, the domain is the set of single-peaked preferences, and the social choice function is strategy-proof.

Lemma 3. Suppose that $n \geq 2, A \subseteq \mathbb{R}$, and $A^{f}$ is an interval of $A$. If $f: \mathcal{S}^{n} \rightarrow A$ is a strategy-proof social choice function, then for all nonempty $H \subset N$ and all $\mathbf{R}^{H} \in \mathcal{S}^{|H|}, O_{-H}^{f}\left(R^{H}\right)$ is a closed interval of $A$.

Proof. In view of Lemma 2, we only need to consider the case in which 
$|H| \geq 2$. First, assume that $|H|=2$ and, without loss of generality, suppose that $H=\{1,2\}$. For any $R^{1} \in \mathcal{S}$, define the $(n-1)$-person social choice function $g_{R^{1}}: \mathcal{S}^{n-1} \rightarrow A$ by setting $g_{R^{1}}\left(R^{2}, \ldots, R^{n}\right)=f\left(R^{1}, \ldots, R^{n}\right)$ for all $\left(R^{2}, \ldots, R^{n}\right) \in \mathcal{S}^{n-1}$. The range of $g_{R^{1}}$ is $O_{-1}^{f}\left(R^{1}\right)$, which, by Lemma 2 , is an interval of $A$. Applying Lemma 2 to $g_{R^{1}}$, it follows that the option set $O_{-H}^{g_{R^{1}}}\left(R^{2}\right)=O_{-H}^{f}\left(R^{1}, R^{2}\right)$ is an interval of $O_{-1}^{f}\left(R^{1}\right)$. Hence, because $O_{-1}^{f}\left(R^{1}\right)$ is an interval of $A, O_{-H}^{f}\left(R^{1}, R^{2}\right)$ is also an interval of $A$. Proceeding by induction on the number of individuals in $H$, the conclusion follows.

Lemma 3 and its proof are due to Barberà and Jackson (1994). The reasoning used to establish this lemma applies whenever a structural property of the range $A^{f}$ of a social choice function $f$ is inherited by non-singelton option sets generated by a single person's preference; i.e., whenever an analogue of Lemma 2 can be established for the domain being considered. When the preferences of the individuals in some group $H$ are fixed at say $\mathbf{R}^{H}$, the social choice only depends on the preferences of the remaining individuals. Thus, fixing $\mathbf{R}^{H}$ defines an $(n-|H|)$-person social choice function $g_{\mathbf{R}^{H}}$ whose arguments are the preferences of the individuals not in $H$. The range of this function is simply the option set generated by the subprofile $\mathbf{R}^{H}$. When $H=\{h\}$, by assumption, this option set exhibits the same structural property as $A^{f}$ or it contains only one alternative. Applying the analogue of Lemma 2 for the domain under consideration to the social choice function $g_{R^{h}}$ implies that any option set of $g_{R^{h}}$ generated by the preference of someone other than $h$ must also either satisfy the same structural property or contain only one alternative. In terms of the original social choice function $f$, it then follows that any option set of $f$ generated by the preferences of two individuals satisfies the structural property or it contains only one alternative. An induction argument on the number of individuals in $H$ then shows that every option set either contains one alternative or it exhibits the structural property assumed for $A^{f}$.

With Lemmas 1, 2, and 3 in hand, it is now a simple matter to show that the tops-only property is satisfied by a strategy-proof social choice function on the domain of single-peaked preferences when the range is an interval.

Theorem 1. Suppose that $n \geq 2, A \subseteq \mathbb{R}$, and $A^{f}$ is an interval of $A$. If $f: \mathcal{S}^{n} \rightarrow A$ is a strategy-proof social choice function, then $f$ satisfies the tops-only property.

Proof. Consider any $\mathbf{R}, \overline{\mathbf{R}} \in \mathcal{S}^{n}$ for which $\tau\left(R^{h}, A^{f}\right)=\tau\left(\bar{R}^{h}, A^{f}\right)$ for all 
$h \in N$. For any $\bar{h} \in N$, it is sufficient to show that if $\tau\left(R^{\bar{h}}, A^{f}\right)=\tau\left(\bar{R}^{\bar{h}}, A^{f}\right)$ and $R^{j}=\bar{R}^{j}$ for all $j \neq \bar{h}$, then $f(\mathbf{R})=f(\overline{\mathbf{R}})$. By Lemma 3 , the option set $O_{\bar{h}}^{f}\left(R^{-\bar{h}}\right)$ is an interval. Further, by Proposition 6, this option set is closed. Because $R^{\bar{h}}$ and $\bar{R}^{\bar{h}}$ are single-peaked with $\tau\left(R^{\bar{h}}, A^{f}\right)=\tau\left(\bar{R}^{\bar{h}}, A^{f}\right)$, they have the same unique maximum on $O_{\bar{h}}^{f}\left(R^{-\bar{h}}\right)$. By Proposition 3, we thus have $f(\mathbf{R})=f(\overline{\mathbf{R}})$.

The proof strategy used to establish Theorem 1 is due to Le Breton and Weymark (1999). This proof strategy can be applied whenever it is the case that if two preferences in the domain have the same most-preferred alternative on the range of the social choice function, then they also have the same most-preferred alternative on any option set generated by the preferences of $n-1$ individuals. For a given domain, whether this property of a social choice function is satisfied depends on the structural properties of the option sets. For any domain for which a version of Theorem 1 applies, the analogue of Lemma 3 would be used to show that the relevant properties of an option set are satisfied. To establish the theorem, there is no loss of generality in supposing that only the preference of one person, say person $\bar{h}$, differs in the two profiles. Because these two preferences have the same top on the range, they must be maximized at the same point $x$ in the option set generated by the other individuals' preferences. By Proposition 3,x must be chosen in both cases, from which the tops-only property immediately follows.

A single-peaked preference is monotonic and, hence, continuous on either side of its peak. This limited form of continuity is used implicitly in the proofs in this section to conclude that a single-peaked preference has a welldefined maximum on any option set. However, a single-peaked preference need not be continuous as it is possible for there to exist alternatives $x, y$, and $z$ such that (i) $x$ is on the other side of the peak from $y$ and $z$, (ii) $y$ is strictly preferred to $x$ which is turn is strictly preferred to $z$, and (3) there is no alternative on the same side of the peak as $y$ and $z$ that is indifferent to $x$. It is readily verified that the proofs used to establish Lemmas 1-3 and Theorem 1 also hold if the domain $\mathcal{S}$ is replaced with the set of continuous single-peaked preferences.

When the range is not an interval, a strategy-proof social choice function $f$ defined on $\mathcal{S}^{n}$ need not satisfy the tops-only property. The problem is that a single-peaked preference on $A$ need not be single-peaked on the range $A^{f}$. Single-peakedness on the range is a property of $f$ that has been used to help prove that the tops-only property is implied by strategy-proofness. 
However, as shown by Barberà and Jackson (1994), if $f$ is strategy-proof, on the subdomain of preferences that are single-peaked on $A^{f}, f$ satisfies the tops-only property. Letting $\hat{f}$ denote the restriction of $f$ to this subdomain, it follows from Proposition 2 that the range of $\hat{f}$ is the same as the range of $f$. Hence, by defining intervals relative to $A^{f}$ (rather than with respect to $A$ ), the arguments used here provide an alternative proof of Barberà and Jackson's theorem. No explicit range assumption is required for $\hat{f}$ because $A^{f}$ is trivally an interval of itself.

\section{Continuous Preferences}

I now assume that $A$ is a metric space. Let $\mathcal{C}$ denote the set of continuous preferences on $A$. Consider a strategy-proof social choice function $f: \mathcal{C}^{n} \rightarrow A$. Barberà and Peleg (1990) have shown that $f$ must be dictatorial provided that the range $A^{f}$ of $f$ contains at least three alternatives. When the domain is $\mathcal{C}$, a dictatorial social choice function need not satisfy the tops-only property. For example, suppose that everyone's preference, including the dictator's, is maximized on $A^{f}$ at both $x$ and $y$. In such a situation, information about preferences other than the tops on the range can be used to break this tie.

Let $\hat{\mathcal{C}}$ be the set of continuous preferences on $A$ that have unique mostpreferred alternatives on the range of $f$ and let $\hat{f}: \hat{\mathcal{C}}^{n} \rightarrow A$ be the restriction of $f$ to this subdomain of preferences. Because $f$ is strategy-proof, so is $\hat{f}$. To prove their theorem, Barberà and Peleg first show that $\hat{f}$ is dictatorial on the subdomain $\hat{\mathcal{C}}$ when $\left|A^{f}\right| \geq 3$. On this subdomain, a dictatorial social choice function satisfies the tops-only property. In this section, I directly show that $\hat{f}$ has the tops-only property regardless of the number of alternatives in its range.

Lemma 4, which is due to Barberà and Peleg (1990), shows that the range $A^{\hat{f}}$ of $\hat{f}$ coincides with $A^{f}$.

Lemma 4. Suppose that $n \geq 2$ and $A$ is a metric space. If $f: \mathcal{C}^{n} \rightarrow A$ is a strategy-proof social choice function, then $A^{\hat{f}}=A^{f}$.

Proof. Because $\hat{\mathcal{C}} \subseteq \mathcal{C}, A^{\hat{f}} \subseteq A^{f}$. Consider any $x \in A^{f}$. By the definition of $\hat{\mathcal{C}}$, there exists a preference $\bar{R} \in \hat{\mathcal{C}}$ such that $\tau\left(\bar{R}, A^{f}\right)=\{x\}$. By Proposition 2, $\hat{f}(\bar{R}, \ldots, \bar{R})=x$. Thus, $x \in A^{\hat{f}}$. Hence, $A^{f} \subseteq A^{\hat{f}}$. 
Because $f$ has a regular domain, by Proposition $5, A^{f}$ is closed. Thus, by Lemma $4, A^{\hat{f}}$ is closed. Furthermore, $\hat{\mathcal{C}}$ is a regular domain for $\hat{f}$. Because $\hat{f}$ inherits strategy-proofness from $f$, it follows from Proposition 6 that all the option sets of $\hat{f}$ must be closed.

I now prove an analogue of Lemma 1 for continuous preferences. Lemma 5 demonstrates that if the social choice function $f$ on the domain $\mathcal{C}$ is strategyproof, then on the subdomain $\hat{\mathcal{C}}$, the option sets of its restriction $\hat{f}$ exhibit the limited form of a tops-only property found in Lemma 1 . That is, if the individuals in subgroup $H$ agree that the same alternative is best on the range, then no other information about their preferences is used to determine the option set generated by their subprofile.

Lemma 5. Suppose that $n \geq 2$ and $A$ is a metric space. If $f: \mathcal{C}^{n} \rightarrow A$ is a strategy-proof social choice function, then for all nonempty $H \subset N$ and all $\mathbf{R}^{H}, \overline{\mathbf{R}}^{H} \in \hat{\mathcal{C}}^{|H|}$ for which $\tau\left(R^{h}, A^{f}\right)=\tau\left(\bar{R}^{k}, A^{f}\right)$ for all $h, k \in H$, $O_{-H}^{\hat{f}}\left(\mathbf{R}^{H}\right)=O_{-H}^{\hat{f}}\left(\overline{\mathbf{R}}^{H}\right)$.

Proof. The lemma is trivial if $\left|A^{f}\right|=1$, so suppose that $\left|A^{f}\right| \geq 2$. The proof is identical to the proof of Lemma 1 (with $\hat{f}$ substituted for $f$ ) up to the point at which it is concluded that there exists a $y \in O_{-H}^{f}\left(\mathbf{R}^{H, \bar{h}-1}\right)$ with $y \notin$ $O_{-H}^{f}\left(\mathbf{R}^{H, \bar{h}}\right)$. As in the proof of Lemma 1, let $\{x\}=\tau\left(R^{h}, A^{f}\right)=\tau\left(\bar{R}^{h}, A^{f}\right)$ for all $h \in H$.

I now show that there exists a preference $R^{*} \in \hat{\mathcal{C}}$ that (i) has its unique top at $y$ and (ii) is maximized on the option set $O_{-H}^{f}\left(\mathbf{R}^{H, \bar{h}}\right)$ at a unique alternative $z$ that is preferred to $y$ by any preference whose most-preferred alternative on the range is at $x$. Because the option set $O_{-H}^{f}\left(\mathbf{R}^{H, \bar{h}}\right)$ is closed and $y \notin O_{-H}^{f}\left(\mathbf{R}^{H, \bar{h}}\right)$, it is easy to construct such a preference. The preference $R^{*}$ is chosen so that it has two local maxima. The first is a global maximum at $y$, which is strictly preferred to the second local maximum, which is located at $x$. Because there exists an open neighbourhood containing $y$ that has an empty intersection with $O_{-H}^{f}\left(\mathbf{R}^{H, \bar{h}}\right)$, such a "double-peaked" preference $R^{*}$ for which $R^{*}$ is maximized on $O_{-H}^{f}\left(\mathbf{R}^{H, \bar{h}}\right)$ at $x$ necessarily exists. ${ }^{9}$ Thus, property (ii) is satisfied with $z=x$. The rest of the proof is identical to the proof of Lemma 1.

The strategy used to prove Lemma 5 is essentially the same as the strategy used to prove Lemma 1 . The only substantive difference is how the preference

\footnotetext{
${ }^{9}$ For a more formal discussion of the construction of $R^{*}$, see Barberà and Peleg (1990).
} 
satisfying properties (i) and (ii) is identified, which is necessarily domain specific.

Next, I need to identify a structural property of the range of $f$ (and, hence, of $\hat{f})$, that is preserved by the non-singleton option sets of the social choice function $\hat{f}$. As noted in the preceding section, for my proof strategy to apply, it is necessary that each preference $R$ in the domain has a unique maximum on any set of alternatives that satisfies the structural property assumed for the range, and this maximum must only depend on the top of $R$ on the range. For any closed strict subset $S$ of the range containing at least two alternatives and any alternative $x \in A^{f} \backslash S$, using constructions similar to the one employed in the proof of Lemma 5 , it is possible to find two preference in $\hat{\mathcal{C}}$ with tops at $x$ that are maximized at different points in $S$. Hence, the only candidate for the required structural property is that of identity. Thus, the analogue to Lemma 2 for the domain $\hat{\mathcal{C}}$ shows that the option set generated by a single individual's preference is either all of the range or a single alternative. ${ }^{10}$

Lemma 6. Suppose that $n \geq 2$ and $A$ is a metric space. If $f: \mathcal{C}^{n} \rightarrow A$ is a strategy-proof social choice function, then for all $h \in N$ and all $R^{h} \in \hat{\mathcal{C}}$, $O_{-h}^{\hat{f}}\left(R^{h}\right)$ is either a singleton or all of $A^{f}$.

Proof. The proof is trival if $\left|A^{f}\right| \leq 2$, so suppose that $\left|A^{f}\right| \geq 3$. By Proposition $6, O_{-h}^{\hat{f}}\left(R^{h}\right)$ is a closed set. Contrary to the lemma, suppose that there exists an $h \in H$ and an $R^{h} \in \mathcal{S}$ such that $O_{-h}^{\hat{f}}\left(R^{h}\right)$ is neither a singleton nor all of $A^{f}=A^{\hat{f}}$. By the definition of $\hat{\mathcal{C}}, \tau\left(R^{h}, A^{f}\right)$ contains a single alternative, say $\gamma$. By Proposition $4, \gamma \in O_{-h}^{\hat{f}}\left(R^{h}\right)$. Hence, there exist $\alpha, \beta \in A^{f}$ such that $\alpha \in O_{-h}^{\hat{f}}\left(R^{h}\right), \alpha \neq \gamma$, and $\beta \notin O_{-h}^{f}\left(R^{h}\right)$. Because $\tau\left(R^{h}, A^{f}\right)=\{\gamma\}$, we must have $\gamma P^{h} \alpha$.

As in the proof of Lemma 5, we can construct a "double-peaked" preference $R^{\alpha} \in \hat{\mathcal{C}}$ with peak at $\beta$ that is maximized on $O_{-h}^{\hat{f}}\left(R^{h}\right)$ at $\alpha$ and a "double-peaked" preference $R^{\gamma} \in \hat{\mathcal{C}}$ with peak at $\beta$ that is maximized on $O_{-h}^{\hat{f}}\left(R^{h}\right)$ at $\gamma$. By Proposition 3 , we have $\hat{f}\left(R^{h} ; R^{\alpha}, \ldots, R^{\alpha}\right)=\alpha$ and $\hat{f}\left(R^{h} ; R^{\gamma}, \ldots, R^{\gamma}\right)=\gamma$. By Lemma $5, O_{h}^{\hat{f}}\left(R^{\alpha}, \ldots, R^{\alpha}\right)=O_{h}^{\hat{f}}\left(R^{\gamma}, \ldots, R^{\gamma}\right)$. Strategy-proofness implies that $R^{h}$ is maximized at $\alpha$ on $O_{h}^{\hat{f}}\left(R^{\alpha}, \ldots, R^{\alpha}\right)$

\footnotetext{
${ }^{10}$ In the latter case, it follows from Proposition 4 that the option set generated by $R^{h}$ is $h$ 's most-preferred alternative on the range.
} 
and at $\gamma$ on $O_{h}^{\hat{f}}\left(R^{\gamma}, \ldots, R^{\gamma}\right)$. Therefore, $\alpha I^{h} \gamma$, contradicting our observation that $\gamma P^{h} \alpha$.

The proof of Lemma 6 differs from that of Lemma 2 in only two respects. First, the alternative $\gamma$ can be chosen to be equal to the top of $R^{h}$ on the range of $\hat{f}$. Second, the construction of the preferences $R^{\alpha}$ and $R^{\gamma}$ are domain specific.

Next, I show that all option sets, not just those generated by a single individual's preference, are either a singleton or all of the range $A^{f}$.

Lemma 7. Suppose that $n \geq 2$ and $A$ is a metric space. If $f: \mathcal{C}^{n} \rightarrow A$ is a strategy-proof social choice function, then for all nonempty $H \subset N$ and all $\mathbf{R}^{H} \in \hat{\mathcal{C}}^{|H|}, O_{-H}^{\hat{f}}\left(R^{H}\right)$ is either a singleton or all of $A^{f}$.

Proof. In view of Lemma 6, we only need to consider the case in which $|H| \geq 2$. First, assume that $|H|=2$ and, without loss of generality, suppose that $H=\{1,2\}$. For any $R^{1} \in \hat{\mathcal{C}}$, define the $(n-1)$-person social choice function $g_{R^{1}}: \hat{\mathcal{C}}^{n-1} \rightarrow A$ by setting $g_{R^{1}}\left(R^{2}, \ldots, R^{n}\right)=\hat{f}\left(R^{1}, \ldots, R^{n}\right)$ for all $\left(R^{2}, \ldots, R^{n}\right) \in \hat{\mathcal{C}}^{n-1}$. The range of $g_{R^{1}}$ is $O_{-1}^{\hat{f}}\left(R^{1}\right)$, which, by Lemma 6 , is either a singleton or all of $A^{f}$. Applying Lemma 6 to $g_{R^{1}}$, it follows that the option set $O_{-H}^{g_{R^{1}}}\left(R^{2}\right)=O_{-H}^{\hat{f}}\left(R^{1}, R^{2}\right)$ is also either a singleton or all of $A^{f}$. If it is a singleton, then for any $\bar{H} \supseteq H, O_{-\bar{H}}^{\hat{f}}\left(R^{\bar{H}}\right)$ must be as well, and the proof is complete. If it is not, then proceeding by induction on the number of individuals in $H$, the conclusion follows.

The property that the option sets exhibit differs in Lemmas 3 and 7, but this does not affect the proof strategy used to establish these results.

Finally, using the same basic proof strategy as was used to prove Theorem 1 , I show that the restriction to the domain $\hat{\mathcal{C}}$ of a strategy-proof social choice function on the domain $\mathcal{C}$ satisfies the tops-only property.

Theorem 2. Suppose that $n \geq 2$ and $A$ is a metric space. If $f: \mathcal{C}^{n} \rightarrow A$ is a strategy-proof social choice function, then $\hat{f}$ satisfies the tops-only property.

Proof. The proof is the same as the proof of Theorem 1 (with $\hat{f}$ substituted for $f$ ) with the following modifications. Lemma 7 is used to conclude that the option set $O_{\bar{h}}^{\hat{f}}\left(R^{-\bar{h}}\right)$ is either a singleton or all of $A^{f}$. Because $R^{\bar{h}}$ and $\bar{R}^{\bar{h}}$ have the same top on the range, in either case it trivially follows that they are maximized at the same point in $O_{\bar{h}}^{\hat{f}}\left(R^{-\bar{h}}\right)$. 


\section{Concluding Remarks}

Recall that a social choice function is nonimposed if its range is equal to the set of alternatives $A$ on which preferences are defined. The set of alternatives $A$ is a product set if it can be written as $A=\prod_{i=1}^{m} A_{i}$ for some $m \geq 2$. For a variety of domains of preferences, the tops-only property has been shown to follow from strategy-proofness when $A$ is a product set and the social choice function is nonimposed. Examples include: (i) the domain of separable quadratic preferences on $A$ when $A_{i}=\mathbb{R}$ for all $i$ (Le Breton and Sen (1995)), (ii) the domain of separable star-shaped preferences on $A$ when $A_{i}=\mathbb{R}$ for all $i$ (Le Breton and Sen (1995)), (iii) the domain of multidimensional singlepeaked preferences when $A$ is a grid; i.e., each set $A_{i}$ consists of a finite number of points with a fixed distance separating adjacent points (Barberà, Gul, and Stacchetti (1993)), and (iv) the domain of separable preferences on $A$ when $A_{i}=\{0,1\}$ for all $i$ (Barberà, Sonneneschein, and Zhou (1991)). ${ }^{11}$ In the latter two examples, it is supposed that preferences are strict, so that no two alternatives are indifferent to each other.

Except for their analogues of Lemmas 1 and 5, Le Breton and Sen's method of proof for the domains of separable quadratic and separable starshaped preferences employs the same basic strategy used to prove the topsonly property in Le Breton and Weymark (1999) and, hence, exhibits many of the features of the proof strategy proposed here. It is possible to also prove the analogues to Lemmas 1 and 5 for these two domains using my method, but the alternative corresponding to $y$ cannot be an arbitrary point lying outside the option set $O_{-H}^{f}\left(\mathbf{R}^{H, \bar{h}}\right)$. My proof strategy can also be used to establish the tops-only property on the other domains described above.

My proof strategy presupposes that every preference in the domain has a unique top on the range of the social choice function. ${ }^{12}$ As has been noted above, when this is not the case, a strategy-proof social choice function need not satisfy the tops-only property. For each individual $h$, strategy-proofness

\footnotetext{
${ }^{11}$ Le Breton and Sen's theorems are implied by the main theorems in Border and Jordan (1983), which characterize the set of strategy-proof social choice functions on the domains of separable quadratic and separable star-shaped preferences that satisfy a unanimityrespecting strengthening of nonimposition. Besides providing an explicit proof of their tops-only theorems, Le Breton and Sen also provide simple proofs of Border and Jordan's theorems.

${ }^{12}$ This assumption is automatically satisfied if all preferences are asymmetric, as in Barberà, Sonneneschein, and Zhou (1991) and Barberà, Gul, and Stacchetti (1993).
} 
requires that the chosen alternative maximizes $h$ 's preference on the option set he faces. If there is more than one alternative in this top set, then a strategy-proof tie-breaking rule must be employed to select among these alternatives. Barberà and Jackson (1994) have examined strategy-proof tiebeaking rules for the domain of single-peaked preferences considered in Section 4 when the range is not an interval of the real line. ${ }^{13}$ They have shown that except for a limited and identifiable set of profiles, strategy-proof tiebreaking rules satisfy the tops-only property.

The proofs presented here rely extensively on the propositions presented in Section 3, and many of these propositions use the assumption that everyone has the same set of admissible preferences in a fundamental way. As a consequence, my proof strategy does not apply if individuals have different sets of admissible preferences, as would be the case if there are private goods. Nevertheless, tops-only theorems have been established in models with private goods by, for example, Sprumont (1991) and Serizawa (1999), but the proofs are model specific. ${ }^{14}$

It is my hope that the proof strategy proposed here will facilitate progress in our understanding of strategy-proof social choice on domains other than the ones considered in this article. As the analysis of the examples in Sections 4 and 5 demonstrates, the proofs of tops-only theorems when there are no private components to preferences need not be model specific, nor need they be particularly complicated.

\section{Acknowlegements}

This article is based on my plenary address to the Association of Public Economic Theory conference held at the Université Paris 1 (Panthéon-Sorbonne), July 4-6, 2002. It has its origins in my presentation to the Conference on New Directions in the Theory of Markets and Games held at the Fields Institute for Research in the Mathematical Sciences, University of Toronto, October 19-23, 1995. No written text was prepared for either of these occasions. The written version was prepared for the Wallis Institute of Political Economy Conference on Game Theory and Social Choice at the University of Rochester, April 16-17, 2004 and the Center of Excellence Project on Research in Economic Systems Symposium on Social Choice Theory, Hitot-

\footnotetext{
${ }^{13}$ See also the related discussion in Barberà and Jackson (1995).

${ }^{14}$ In Serizawa's article, private goods are used to finance the production of a public good.
} 
subashi University, July 19-20, 2004. I have greatly benefitted from my joint research on strategy-proofness with Michel Le Breton. I have also benefitted from discussions and/or correspondence with Shurojit Chatterji, John Duggan, Lars Ehlers, Arunava Sen, and Lin Zhou.

\section{References}

Barberà, S., 1983. Strategy-proofness and pivotal voters: A direct proof of the Gibbard-Satterthwaite theorem. International Economic Review 24, 423-417.

Barberà, S., 2001. An introduction to strategy-proof social choice functions. Social Choice and Welfare 18, 619-653.

Barberà, S., Gul, F., Stacchetti, E., 1993. Generalized median voting schemes and committees. Journal of Economic Theory 61, 262-289.

Barberà, S., Jackson, M., 1994. A characterization of strategy-proof social choice functions for economies with pure public goods. Social Choice and Welfare 11, 241-252.

Barberà, S., Jackson, M., 1995. Strategy-proof exchange. Econometrica 63, 51-87.

Barberà, S., Massó, J., Neme, A., 1997. Voting under constraints. Journal of Economic Theory 76, 298-321.

Barberà, S., Peleg, B., 1990. Strategy-proof voting schemes with continuous preferences. Social Choice and Welfare 7, 31-38.

Barberà, S., Sonneneschein, H., Zhou, L., 1991. Voting by committees. Econometrica 59, 595-609.

Border, K. C., Jordan, J. S., 1983. Straightforward elections, unanimity and phantom voters. Review of Economic Studies 50, 153-170.

Ching, S., 1997. Strategy-proofness and "median voters". International Journal of Game Theory 26, 473-490.

Dutta, B., Peters, H., Sen, A., 2002. Strategy-proof probabilistic mechanisms in economies with pure public goods. Journal of Economic Theory 106, 392-416.

Ehlers, L., Peters, H., Storcken, T., 2002. Strategy-proof probabilistic decision schemes for one-dimensional single-peaked preferences. Journal of Economic Theory 105, 408-434.

Gibbard, A., 1973. Manipulation of voting schemes: A general result. Econometrica 41, 587-601. 
Gibbard, A., 1977. Manipulation of voting schemes that mix voting with chance. Econometrica 45, 665-681.

Laffond, G., 1980. Révelation des preferences et utilités unimodales. Thèse pour le doctorat, Laboratoire d'Econométrie, Conservatoire National des Arts et Métiers.

Le Breton, M., Sen, A., 1995. Strategyproofness and decomposability: Weak orderings. Discussion Papers Series in Economics No. 95-04, Indian Statistical Institute, Delhi Centre.

Le Breton, M., Weymark, J. A., 1999. Strategy-proof social choice with continuous separable preferences. Journal of Mathematical Economics 32, 4785.

Moulin, H., 1980. On strategy-proofness and single peakedness. Public Choice $35,437-455$.

Satterthwaite, M. A., 1975. Strategy-proofness and Arrow's conditions: Existence and correspondence theorems for voting procedures and social welfare functions. Journal of Economic Theory 10, 187-217.

Satterthwaite, M. A., Sonnenschein, H., 1981. Strategy-proof allocation mechanisms at differentiable points. Review of Economic Studies 48, 587597.

Serizawa, S., 1999. Strategy-proof and symmetric social choice functions for public good economies. Econometrica 67, 121-145.

Sprumont, Y., 1991. The division problem with single-peaked preferences: A characterization of the uniform allocation rule. Econometrica 59, 509-519.

Sprumont, Y., 1995. Strategyproof collective choice in economic and political environments. Canadian Journal of Economics 48, 68-107. 


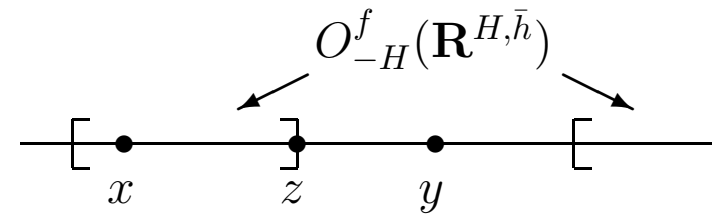

Figure 1

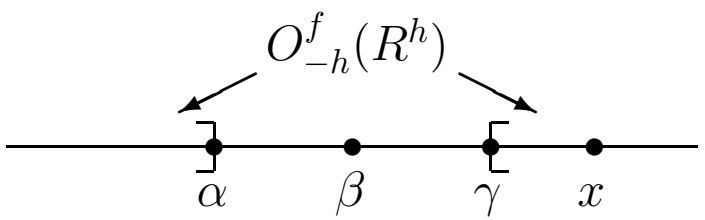

Figure 2 